\title{
Anesthetic Impacts on the Oculocardiac Reflex: Evidence from a Large, Observational Study
}

This article was published in the following Dove Press journal: Clinical Ophthalmology

\author{
Robert W Arnold (iD) \\ Stephen Jansen ${ }^{2}$ \\ Joseph C Seelig ${ }^{2}$ \\ Mikhail Glasionov ${ }^{3}$ \\ Russell E Biggs ${ }^{2}$ \\ Brion Beerle ${ }^{2}$
}

'Alaska Children's EYE \& Strabismus, Anchorage, AK, 99508, USA; ${ }^{2}$ Chugach Anesthesia Group, Anchorage, AK, USA; ${ }^{3}$ Department of Anesthesia, Alaska Regional Hospital, Anchorage, AK, USA

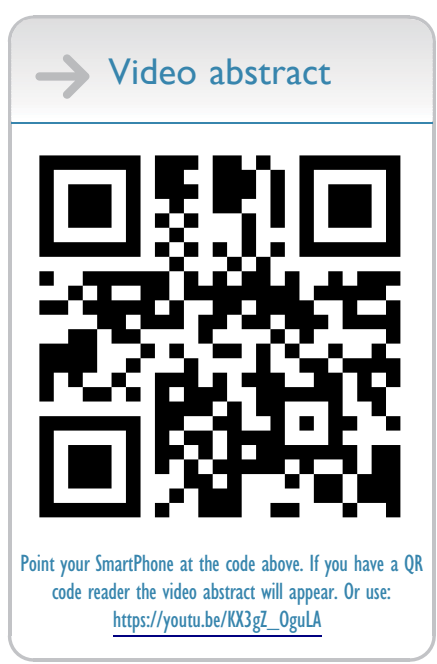

Correspondence: Robert W Arnold Alaska Blind Child Discovery, Alaska Children's EYE \& Strabismus, 3500 Latouche \#280, Anchorage, AK, 99508, USA

Tel + I $90756 \mathrm{I}-1917$

$\mathrm{Fax}+1907563-5373$

Email eyedoc@alaska.net
Background: The oculocardiac reflex (OCR) is a sudden vagal bradycardia that can be elicited by traction on an extraocular muscle. Bradycardia is highly variable from case to case necessitating a large sample size to observe small to moderate impact on OCR. While the surgeon's tissue manipulation has immediate impact on OCR and individual patients may have some proclivity to OCR, we sought to characterize the impact on OCR by the anesthesiologist. Methods: From 1992 to 2019, during routine, community outpatient general anesthetic strabismus surgery, oculocardiac reflex was prospectively observed utilizing a uniform 10second, 200 gram square wave tension on each extraocular muscle. Anesthetic parameters were recorded and analyzed with double-cohort design and non-parametric statistics and correlations. We define \%OCR as the maximally tension-altered heart rate and a percent of stable pre-tension heart rate.

Results: The median (IQR) confidence intervals OCR for 2527 initial cases was $89 \%$ (67\% to $97 \%$ ) without anticholinergic, and $99 \%$ (95\% to $100 \%)$ in 165 patients with anticholinergic. OCR was $81 \%(62 \%$ to $96 \%)$ in 1034 with opioids and to $75 \%(60 \%$ to $95 \%)$ in 59 with dexmedetomidine and in 189 with IV dexamethasone to $72 \%(56 \%$ to $92 \%)$ There was a significant $(\mathrm{p}<0.01 \mathrm{Kruskal-Wallis)}$ impact on OCR by various opioids, muscle relaxants and inhalational agents. Linear regression showed significant inhibitory impact on OCR by increased inhalational agent depth and by lower exhaled $\mathrm{CO}_{2}$.

Conclusion: The anesthesiologist can block OCR with sufficient anticholinergics, deeper inhalational agents and robust ventilation, and can augment OCR with opioids, dexmedetomidine and apparently also with dexamethasone.

Clinical Trials Registry: NCT04353960.

Keywords: oculocardiac reflex, trigeminovagal, strabismus surgery, opioids, dexmedetomidine, dexamethasone, anticholinergics

\section{Plain Language Summary}

Several previous smaller studies have addressed certain aspects of anesthetic impact on the typical bradycardia during strabismus surgery called the oculocardiac reflex (OCR). Due to a wide inter-patient variability in OCR, large studies are needed to confirm findings. We reviewed over 2500 patients confirming that OCR is augmented by opioids, dexmedetomidine, rocuronium and dexamethasone while deeper inhalational agents, hypocapnia and anticholinergics reduce OCR.

\section{Introduction}

The oculocardiac reflex (OCR) is triggered by manipulation of the eye or orbit resulting in a change in heart rate which is usually a vagal bradydysrythmia. ${ }^{1,2}$ OCR 
as a trigeminovagal reflex is commonly observed in children and adults undergoing strabismus surgery. ${ }^{3}$ Of several common vagal reflexes including Valsalva maneuver and diving response, surgical OCR has the widest case-to-case variability ranging from profound bradycardia to moderate tachycardia. $^{4}$

The surgeon's influence is immediate and profound. ${ }^{5}$ The critical amount of extraocular muscle (EOM) tension, ${ }^{6}$ the type of EOM, and gentle repeat stimuli allows the surgeon to exert an inhibitory influence on OCR. ${ }^{7}$

The patients themselves may provide some predictability relative to $\mathrm{OCR} .{ }^{8} \mathrm{~A}$ given patient's initial OCR is a good predictor of their second OCR in a 2-muscle case. ${ }^{9}$

A large sample size is needed to detect differences due to a typical mean and standard deviation (S.D.) OCR effect. ${ }^{8}$ Some details regarding the impact of the anesthesiologist on OCR have been studied in smaller, underpowered clinical trials that lack EOM tension control, ${ }^{10}$ so we sought to confirm anesthetic impacts and determine which are actually the most important. Therefore, we reviewed results from our ongoing, observational trial that, for almost three decades, has diligently recorded pertinent patient and anesthetic variables each with prospective, uniform extraocular tension as the elicitation of OCR.

\section{Methods}

This prospective, observational cross-sectional, doublecohort study has Institutional Review Board approval from Providence Hospital (Providence Alaska Medical Center). It complies with the Health Insurance Portability and Accountability Act of 1996 and the Declaration of Helsinki. Due to the strict, observational nature recording traditional monitor data from routine strabismus surgery, the institutional review board waived the requirement for signed, informed consent. The clinical trials identifier is NCT04353960. De-identified data from 1992 to 2019 can be viewed indefinitely from the study website: http://www. abcd-vision.org/OCR/OCR-anesthetic\%20de-identified $\%$ 20data.pdf.

This is an observational trial of conventional community out-patient general anesthesia for conventional strabismus surgery in children and adults (Figure 1). Consecutive cases of strabismus surgery in children and adults were monitored with electrocardiograph and prospectively-determined, uniform tension stimulus on gently isolated extraocular rectus muscles. Re-operations and cases with anticholinergic medications were identified.
The choice of anesthetic technique is at the discretion of the anesthesiologist with a surgeon request to avoid anticholinergic medications unless specifically indicated. Patients general health is listed as ASA (American Society of Anesthesiologists) 1 or 2. Patients with orbital block or pacemaker or ASA $>2$ were excluded. Intravenous access was attained pre-operatively in the all adults and teenagers while younger children had intravenous placement after mask induction. Some patients had pre-operative sedation with oral or intravenous midazolam while others had intranasal dexmedetomidine. ${ }^{11}$ Some patients had induction with just inhalational agents with or without nitrous oxide, while others had intravenous propofol, opioids and/or dexmedetomidine during induction. Heart rate by electrocardiograph, fingertip pulse oxymetry, blood pressure and inhaled/exhaled carbon dioxide and inhalational agent concentrations were monitored throughout the procedure. Some patients had intravenous muscle relaxants to aid in induction and airway placement. Most patients had laryngeal mask airway while others, at the choice of the anesthesiologist, had endotracheal intubation.

Many patients were maintained with inhalational anesthetics of sufficient depth ${ }^{12}$ to avoid extremity movement or blepharospasm with ocular manipulation, but light enough to eventually afford spontaneous ventilation; the ventilation and agent concentrations were often changed from the first extraocular muscle to the second. Dexamethasone was added in some patients hoping to reduce post-operative nausea and vomiting.

The stimulus on an extraocular muscle for oculocardiac reflex has been the same for each case. An extraocular rectus muscle is gently approached and hooked with a thin Jameson hook. The anesthesiologist ensures stable pre-stimulus heart rate, blood pressure and oxygen saturation. Then a small, sterile tension gauge is attached to the Jameson hook, and a 10 -second, square wave tension of 200 grams is placed on the isolated rectus muscle. The greatest change is then recorded and evaluated as a percent of pre-stimulus, stable heart rate with values less than 100 (5\% to $99 \%)$ being bradycardia, values greater than 100 (101\% to $151 \%)$ being tachycardia and $100 \%$ representing no change. For example, a $25 \%$ heart rate drop from a baseline of $120 \mathrm{~b}$. p.m. to 90 b.p.m. during EOM tension would be reported as an OCR of $75 \%$. If more than one muscle was operated in a given case, two or more oculocardiac recordings could be taken for that patient, that day. A video example of profound oculocardiac reflex can be viewed (Supplementary Video) . 

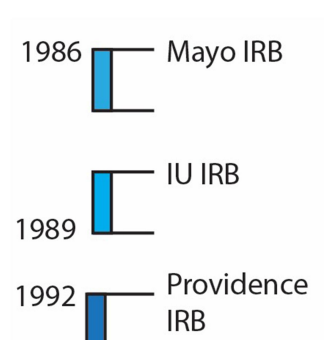

1995

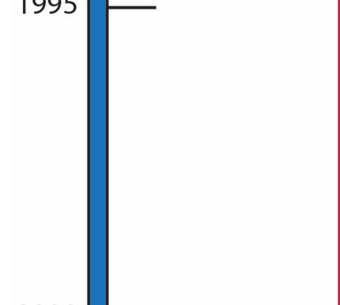

2000

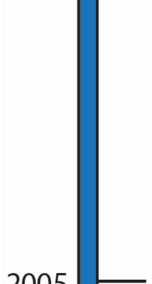

2005

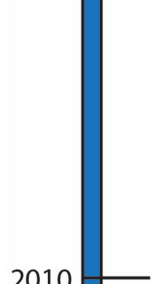

2010

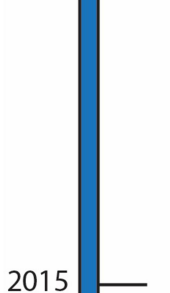

2015

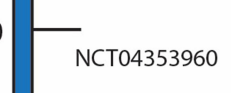

Strabismus Patients

Twins

OCR-prone?

Controlled depth reduction

Topical profound
5 Vagal maneuvers

Iris color

Intraglossal atropine

EOM tension

Re-op BP

Opioids augment

dexmedetomidine nasal

dexmedetomidine IV

Topical proparacaine

BIS Narcotrend

Re-op relatives race

Figure I Timeline of the Alaska OCR Study. Uniform elicitation of oculocardiac reflex (OCR) consistent with routine strabismus surgery has employed I0-second, 200gram square wave tension on rectus extraocular muscles (EOM). Years are to the left and numbers of patients are listed at the bottom. The Alaska study mirrored two prior studies at Mayo Clinic and Indiana University. Publications and presentations of OCR data are listed to the right of the timeline. 


\section{Statistical Methods}

Sample Size: To detect a 15\% difference between expected means of $80 \%$, standard deviation (S.D.) of $20 \%$, power 0.8 and alpha 0.5 , a sample size of 28 is needed. The impact of intravenous atropine on OCR is about a $15 \%$ difference. To detect a 5\% difference, a sample size of 251 is needed. The difference in OCR between adults and children is about 5\%. To detect a 3\% difference, a sample size of 697 is needed. The selection of different extraocular muscle types produces about a 3\% change. The majority of articles in the literature are underpowered for the widely variable human oculocardiac reflex; only four other studies have a sample size over 200 patients. ${ }^{8}$ Our database is sufficiently powered to detect difference of $3 \%$ or less between groups.

The degree of normal distribution of the cardiac rate change is assessed with kurtosis and skewness. Non- parametric comparison of non-normally distributed continuous data are compared with Mann-Whitney test (2-samples) and the Kruskal-Wallis test ( $>2$ samples). Matched continuous datasets were analyzed with linear regression and Pearson's Product Moment Correlation with $\mathrm{p}<0.01$ considered significant.

\section{Results}

Table 1 includes the oculocardiac reflex recordings and anesthetic influences from 2527 original cases separated from those with anticholinergic medication (Figure 1). The frequency distribution of oculocardiac values (Figure 2) has been widely (range $5 \%$ to $151 \%$ ) but not normally distributed with kurtosis and skewness, ${ }^{8}$ so data are analyzed using non-parametric tests. The median oculocardiac reflex from the first muscle tested $(85 \%)$ did not differ from the oculocardiac elicited when a second muscle was

Table I The Influence on the Oculocardiac Reflex by Various Anesthetic Parameters. Nonparametric Values Centered on the Median with First and Third Quartiles and the 5th and 95th Percentiles. The Mean and Standard Deviation (S.D.) are Given as Well as Proportion with More Than 20\% Drop in Heart Rate (>20drop) and More Than 50\% Drop in Heart Rate (>50drop). \%OCRI and \% OCR2 are from the First and Second Extraocular Muscle During Each Strabismus Case Each Elicited by a 10-Second, 200 Gram Tension on a Gently Isolated Rectus Muscle. OCR is Expressed as the Maximal Percent Change from a Stable, Pre-Tension Heart Rate. Impacts are Grouped by Anticholinergic, Opioids, Propofol, Dexamethasone and Dexmedetomidine, Muscle Relaxants and Inhalational Agents

\begin{tabular}{|c|c|c|c|c|c|c|c|c|c|c|c|c|}
\hline Influence & Number & Minimum & $5 \%$ & QI & Median & Q3 & $95 \%$ & Maximum & Mean & S.D. & $>20$ drop & $>50$ drop \\
\hline \%OCRI & 2527 & 5 & 39 & 67 & 85 & 97 & 102 & $|5|$ & 80 & 21 & 41 & 10 \\
\hline \%OCR2 & 1526 & 5 & 42 & 69 & 86 & 98 & 102 & 182 & 81 & 21 & 39 & 8 \\
\hline Anticholinergic & 165 & 9 & 70 & 95 & 99 & 100 & 103 & 112 & 95 & 12 & 6 & 0 \\
\hline Any opioid & 1034 & 6 & 27 & 62 & 81 & 96 & 102 & 122 & 76 & 23 & 49 & 14 \\
\hline Fentanyl & 845 & 6 & 29 & 63 & 81 & 96 & 102 & 122 & 76 & 23 & 48 & 13 \\
\hline Hydromorphone & 73 & 20 & 37 & 61 & 76 & 93 & 100 & $|2|$ & 75 & 22 & 56 & 15 \\
\hline Remifentanil & 53 & 13 & 18 & 48 & 78 & 92 & 105 & 122 & 70 & 28 & 52 & 26 \\
\hline Morphine & 44 & 15 & 48 & 65 & 86 & 96 & 107 & 110 & 80 & 22 & 36 & 9 \\
\hline Sufentanil & 19 & 14 & 14 & 56 & 84 & 96 & 103 & 103 & 74 & 28 & 42 & 16 \\
\hline Propofol & 470 & 6 & 19 & 64 & 87 & 98 & 102 & 129 & 78 & 25 & 41 & 14 \\
\hline Dexamethasone & 189 & 6 & 16 & 56 & 72 & 92 & 100 & 118 & 70 & 25 & 59 & 20 \\
\hline Dexmedetomidine & 59 & 8 & 28 & 60 & 75 & 95 & 101 & 113 & 72 & 24 & 47 & 18 \\
\hline Atracurium & 31 & 14 & 26 & 74 & 92 & 99 & 111 & 129 & 83 & 24 & 39 & 10 \\
\hline Cisatracurium & 11 & 9 & 9 & 74 & 97 & 99 & 100 & 100 & 81 & 30 & 27 & 18 \\
\hline Mivacurium & 160 & 8 & 20 & 63 & 82 & 96 & 101 & $|3|$ & 76 & 24 & 47 & 13 \\
\hline Rocuronium & 72 & 10 & 17 & 63 & 78 & 97 & 100 & 122 & 75 & 26 & 53 & 17 \\
\hline Succinylcholine & 74 & 26 & 45 & 82 & 96 & 100 & 105 & 119 & 89 & 18 & 23 & 5 \\
\hline Vecuronium & 51 & 6 & 16 & 60 & 88 & 94 & 100 & 103 & 75 & 26 & 43 & 18 \\
\hline Desflurane & 190 & 8 & 49 & 72 & 94 & 99 & 107 & 122 & 85 & 20 & 31 & 6 \\
\hline Halothane & 321 & 15 & 54 & 75 & 87 & 97 & 101 & $13 \mid$ & 83 & 17 & 33 & 4 \\
\hline Isoflurane & 323 & 6 & 22 & 66 & 89 & 98 & 102 & 129 & 80 & 24 & 38 & 12 \\
\hline Sevoflurane & 1319 & 5 & $4 I$ & 67 & 84 & 97 & 102 & $15 \mid$ & 80 & 21 & 44 & 10 \\
\hline
\end{tabular}




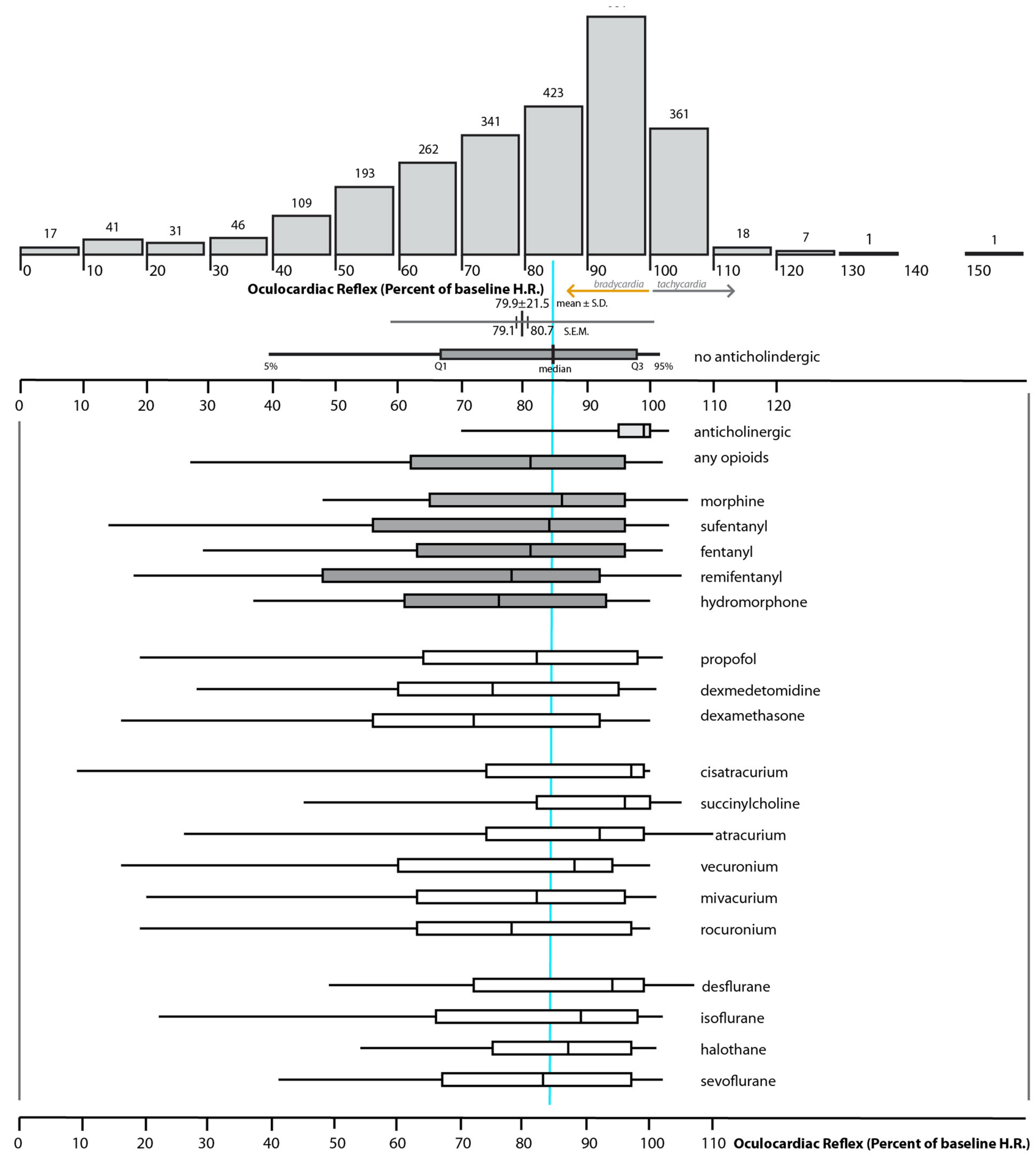

Figure 2 Frequency distribution of oculocardiac reflex showing anesthetic impacts. Oculocardiac reflex (OCR) defined as the maximally tension altered heart rate as a percent of stable, pre-tension baseline heart rate (H.R.). Uniform stimulus is 10 -second, 200 gram tension on an extraocular rectus muscle. Impacts of various anesthetic agents indicated by box and whisker plots defining interquartile range, $95 \%$ range with bar to define median. S.D. is standard deviation and S.E.M. is standard deviation of the mean.

tested during the same case $(86 \%$, Mann-Whitney $\mathrm{z}=1.69$, $\mathrm{p}=0.09$ ). Figure 3 shows the relationship between initial OCR and second OCR on 1524 two-muscle cases. An additional 169 cases had anticholinergic which significantly reduced the median OCR from $85 \%$ to $99 \%$ (Mann-Whitney $\mathrm{z}=11.2, \mathrm{p}<0.01$ ). Of note, we excluded from this paper but recently reported in a different paper ${ }^{8}$ oculocardiac recordings in 446 re-operation cases from 


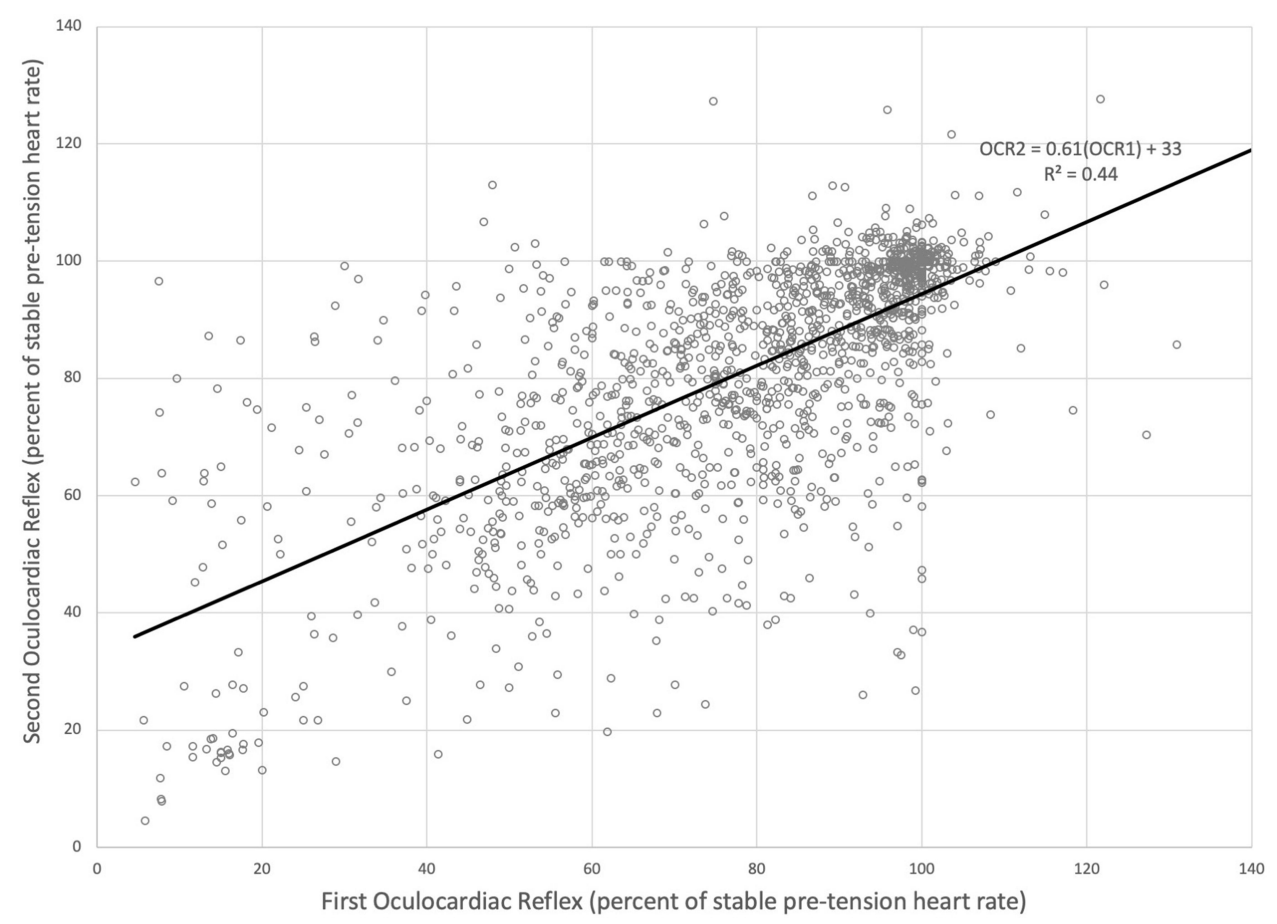

Figure 3 Linear correlation between first and second oculocardiac reflex (OCR). OCR is defined as the tension-modified heart rate as a percent of stable baseline elicited by uniform 10-second, 200 gram tension on an extraocular muscle in 1524 initial unique strabismus adult and pediatric patients with no anticholinergic.

323 of the patients. During this phase of the Alaska OCR study, we have recorded data from 2527 original +154 anticholinergic cases +415 re-operations +15 anticholinergic re-operations +5 blocks totalling 3116 strabismus surgery cases (Figure 1).

Patients ranged in age from 0.2 to 93 years with mean 15.6 years, median 6.5 years, standard deviation 19 years. The patient weights ranged from 4 to 250 kilograms with mean 38.7, standard deviation 33 and median 22 kilograms.

Patients receiving some form of opioid had a significantly greater oculocardiac reflex (median $81 \%$ compared to $85 \%$ for those without opioid; MannWhitney $z=7.0, p<0.01$ ). Compared to no opioid, the median impact of five different opioids was significant (Kruskal-Wallis $\left.\mathrm{X}^{2}(5)=53, \mathrm{p}<0.01\right)$ with morphine having least impact (median 86\%) remifentanil (median 78\%) and hydromorphone (median 76\%) having the most impact.

Patients who received dexmedetomidine had substantially more oculocardiac reflex than those with none (median 75\%; Mann-Whitney $z=3.22, p<0.01)$. An augmented oculocardiac reflex was also associated with intravenous dexamethasone compared to none (median $72 \%$, MannWhitney $\mathrm{z}=5.8, \mathrm{p}<0.01)$. The augmenting impact of dexamethasone persisted independent of opioid, inhalational agent, nitrous oxide, expired carbon dioxide and age. Propofol was not associated with a change in oculocardiac reflex compared to no propofol (median $87 \%$, Mann-Whitney $\mathrm{z}=0.25, \mathrm{p}=0.81$ ).

Six different intravenous muscle relaxants had an impact on median oculocardiac reflex with cisatracurium and succinyl choline decreasing OCR and rocuronium augmenting the reflex (Kruskal-Wallis $X^{2}(6)=29$, $\mathrm{p}<0.01)$. There was a significant difference in median oculocardiac reflex associated with different inhalational agents (Kruskal-Wallis $\mathrm{X}^{2}(4)=18, \mathrm{p}<0.01$ ).

Table 2 shows data from linear regressions between oculocardiac reflex and exhaled gas concentrations. There was a reduction in the initial oculocardiac reflex associated with higher end-tidal concentrations of halothane, isoflurane and sevoflurane. OCR was reduced by lower end-tidal carbon dioxide concentration but unchanged by different levels of nitrous oxide used during induction and/or maintenance inhalational anesthesia.

The difference between second and initial OCR in each case was correlated (Figure 3) with differences between second and initial gas concentrations. Lighter levels of inhaled isoflurane, halothane and sevoflurane 
Table 2 Linear Correlations Between Oculocardiac Reflex as a Percent of Baseline Heart Rate Compared to Exhaled Anesthesia Gas Concentrations. The Ordinate (y) is a Function of Constant a (with 95\% Confidence Intervals), The Abscissa x Gas Concentration and a Constant b Percent Heart Rate with 95\% Confidence Intervals. N is Number of Cases, the Pearson Product Correlation Value $r$ and Probability $\mathrm{P}$ are Given. OCRI is the Initial Oculocardiac Reflex and $\triangle \mathrm{OCR}$ is the Difference Subtracting OCRI from OCR2 in TwoMuscle Cases. $\mathrm{CO}_{2}$ is Carbon Dioxide and $\mathrm{N}_{2} \mathrm{O}$ is Nitrous Oxide

\begin{tabular}{|c|c|c|c|c|c|c|}
\hline y & $\mathbf{a}$ & $\mathbf{x}$ & $+\mathbf{b}$ & $\mathbf{n}$ & $\mathbf{p}$ & Product-Moment Correlation \\
\hline OCRI & $-0.13(-0.22,-0.04)$ & $\mathrm{CO}_{2} \mathrm{mmHg}$ & $85.5(81.6,89.3)$ & 1428 & $<0.01$ & $r(2384)=-0.06$ \\
\hline OCRI & $-1.39(-3.96,1.16)$ & desflurane \% Et & $85.2(79.8,90.7)$ & 190 & 0.12 & $r(164)=0.12$ \\
\hline OCRI & $-6.4 I(-12.5,-0.31)$ & halothane \% Et & $82.4(80.1,84.7)$ & 321 & 0.01 & $r(3 \mid 6)=0.14$ \\
\hline OCRI & $-7.04(-14.1,0.06)$ & isoflurane \% Et & $79.5(76.1,82.8)$ & 323 & 0.04 & $r(316)=0.11$ \\
\hline OCRI & $-2.15(-3.67,-0.64)$ & sevoflurane \% Et & $77.8(76.2,70.4)$ & 1319 & $<0.01$ & $r(1182)=0.11$ \\
\hline OCRI & $0.07(-0.04,0.19)$ & $\mathrm{N}_{2} \mathrm{O} \%$ & $77.9(71.5,84.2)$ & 915 & 0.22 & $r(9 \mid 3)=0.04$ \\
\hline$\triangle O C R$ & $1.35(0.49,2.21)$ & $\Delta$ agent $\%$ & $3.14(2.22,4.05)$ & 1524 & $<0.01$ & $r(1370)=0.08$ \\
\hline$\triangle \mathrm{OCR}$ & $0.03(-0.05,0.12)$ & $\Delta \mathrm{CO}_{2} \mathrm{~mm} \mathrm{Hg}$ & $2.83(1.91,3.75)$ & 1428 & 0.45 & $r(1425)=0.02$ \\
\hline$\triangle \mathrm{OCR}$ & $0.95(-0.70,2.59)$ & $\Delta$ desflurane & $0.49(-3.0,4.0)$ & 190 & 0.26 & $r(70)=0.14$ \\
\hline$\triangle O C R$ & I3.I $(7.6, \mid 8.6)$ & $\Delta$ halothane & $3.90(1.8,5.9)$ & 321 & $<0.01$ & $r(239)=0.29$ \\
\hline$\triangle O C R$ & $9.88(4.5,15.2)$ & $\Delta$ isoflurane & $5.69(3.18,8.19)$ & 323 & $<0.01$ & $r(2 I I)=0.24$ \\
\hline$\triangle O C R$ & I.56 $(0.33,2.78)$ & $\Delta$ sevoflurane & $2.78(1.49,4.06)$ & 1319 & 0.01 & $r(699)=0.09$ \\
\hline
\end{tabular}

produced more oculocardiac reflex, but changes in expired $\mathrm{CO}_{2}$ and nitrous oxide has no significant effect on change in OCR.

\section{Discussion}

Over almost three decades in this observational study of oculocardiac reflex during strabismus surgery with uniformly elicited tension on extraocular rectus muscles, we found a wide case-to-case variability of bradycardia. There is a strong, but not perfect correlation between first and second OCR within the same case even with identical surgical stimulus. Patients may have some limited tendency toward the trigeminovagal reflex. ${ }^{8}$ Variations in various anesthetic agents throughout the course of the case likely influences OCR variability. Our study is observational with anesthetic protocol at the choice of each given anesthesiologist.

The Alaska OCR Study over 28 years (Figure 1) has identified that OCR can be augmented by opioids ${ }^{13}$ and by dexmedetomidine ${ }^{14}$ whereas OCR can be effectively blocked by intraglossal atropine if intravenous access in not available. ${ }^{15}$ Our ongoing study countered one of the larger sample size prior studies ${ }^{16}$ that had suggested iris color influenced OCR. ${ }^{17}$ We studied extraocular muscle tension noting more OCR with inferior than lateral rectus muscle, more OCR with 200 and 400 gram tension than $50 \mathrm{gm}$ tension and that the OCR fatigued if repeat tension quickly followed the first pull. ${ }^{7}$ The Alaska OCR Study found that interoperative brain wave monitors were only minimally predictive of OCR. ${ }^{12}$ Intraoperative OCR is less profound that the bradycardia induced by scleral depressed, indirect ophthalmoscopic exams in infants screened for retinopathy of prematurity (ROP). ${ }^{18}$ Recently, the Alaska ROP study investigated how aspects of the individual patient influenced OCR; adults have less percent OCR than children, race mattered with whites having more bradycardia, the original surgery was minimally predictive of re-operations and relatives did not share OCR proclivity. ${ }^{8}$ We also confirmed that the initial OCR is a good predictor of subsequent OCR in cases with more than one muscle manipulated. ${ }^{19}$

As we initially observed earlier in our observational study, ${ }^{13}$ and was later confirmed with a randomized trial, ${ }^{20}$ opioids augment the oculocardiac reflex. The impact of fast-acting opioids ${ }^{21}$ is of a similar magnitude but opposite to atropine's blocking impact. We confirmed our prior observations that uncovered an augmenting impact of dexmedetomidine on oculocardiac reflex whether delivered nasally pre-op ${ }^{11}$ or intravenously. ${ }^{14}$ Mizrak, et al, on the other hand, found that dexmedetomidine plus ketamine decreased OCR. ${ }^{22}$

We found minimal difference on oculocardiac reflex during general anesthesia influenced by the presence of propofol or nitrous oxide. In patients with fentanyl and rocuronium, ketamine was associated with less OCR than propofol. ${ }^{23}$ Propofol-ketamine produced more OCR but less post-operative nausea and vomiting than sevoflurane - 
nitrous oxide anesthesia ${ }^{24,25}$ or with isoflurane-nitrous anesthesia. $^{26}$

We found that dexamethasone as an antiemetic was associated with augmented oculocardiac reflex. This effect persisted independent of opioid, inhalational agents, expired carbon dioxide and age. In another report, OCR was common $(65 \%)$ in strabismus surgery with dexamethasone and ondansetron. ${ }^{27}$

There was a trend for reduced oculocardiac reflex with deeper inhalational anesthesia - at least with isoflurane, halothane and sevoflurane. This concentration effect occurred during the initial OCR and also the difference in agent concentration impacting the difference between first and second measured OCR in the case. Intentional deep inhalational anesthesia and adequate ventilation reduced OCR in a small series compared to historical controls. ${ }^{28}$ Karaman et $\mathrm{al}^{29}$ and $\mathrm{Yi}$ et $\mathrm{al}^{30}$ found less OCR with deeper anesthesia monitored by BIS brain wave monitoring. Using uniform elicitation of OCR, we found minimal impact of BIS and Entropy monitor readings on OCR. ${ }^{12}$

In our observation of muscle relaxants, rocuronium was associated with more OCR while succinyl choline had less, just opposite to Karanovic's smaller randomized study. ${ }^{31}$ In a small, randomized study, atracurium produced more OCR than pancuronium. ${ }^{32}$

The strength of our study is meticulous, uniform stimulus of OCR representing typical strabismus surgery, uniform continuous data criteria that enhance the statistical power over categorical data. We included a large number of children and adults; adults are known to have less bradycardia with strabismus surgery extraocular muscle tension than do children. ${ }^{8}$ The sample size is large enough to detect small differences between groups. A weakness is that lack of a randomized, double-blind clinical trial with uniformly defined anesthetic protocol. We note that the literature is plush with randomized clinical trials on OCR that are underpowered to adequately address a variable as widely distributed as OCR. While there are recognized differences in anesthetic care over three decades, there are probably also unknown differences for which a bias may have been introduced. Our study is generalizable to community, outpatient strabismus surgery. It should be noted that several medications were studied, but this is not a dose-response study.

With vigilant anesthetic maintenance of premanipulation blood pressure, oxygen saturation and pulse, no adverse sequelae or admission to intensive care prompted by oculocardiac reflex was observed during the three decade course of this observational study.

\section{Conclusion}

The anesthesiologist has great impact on the oculocardiac reflex during strabismus surgery in adults and children. OCR can be blocked by adequate anticholinergic medication but augmented, to a similar degree, with certain opioids and dexmedetomidine. We found that deeper inhalational agents had a small reduction in OCR while hypercapnia tended to increase OCR. Propofol and nitrous oxide had minimal impact on OCR. We also noted increased OCR with dexamethasone used as nausea prophylaxis; we hope others will consider a randomized trial to confirm or refute these findings.

\section{Abbreviations}

ASA, American Society of Anesthesiologists; b.p.m, beats per minute; $\mathrm{CO}_{2}$, carbon dioxide; $\mathrm{N}_{2} \mathrm{O}$, nitrous oxide; OCR, oculocardiac reflex; S.D., standard deviation.

\section{Data Sharing Statement}

Data Access: http://www.abcd-vision.org/OCR/OCRanesthetic\%20de-identified\%20data.pdf.

\section{Disclosure}

Dr. Arnold is President of Glacier Medical Software that markets cloud-based NICU software ROP Check. Dr. Arnold is President of PDI Check that makes a vision screening game for Nintendo 3DS. Dr. Arnold coordinates the Alaska Blind Child Discovery that has received discounted vision screen technology from several vendors. Dr. Arnold is an investigator and protocol developer for the NIH-supported Pediatric Eye Disease Investigator Group. Dr Robert Arnold has a patent pending for PDI Check; and he serves on non-paid advisory boards for plusoptiX, GoCheck Kids, Adaptica and iScreen amblyopia screening. The authors report no other conflicts of interest in this work.

\section{References}

1. vanBrocklin $\mathrm{M}$, Hirons $\mathrm{R}$, Yolton $\mathrm{R}$. The oculocardiac reflex: a review. J Amer Optometric Assoc. 1982;53(5):407-413.

2. Meuwly C, Chowdhury T, Sandu N, Reck M, Erne P, Schaller B. Anesthetic influence on occurrence and treatment of the trigemino-cardiac reflex: a systematic literature review. Medicine (Baltimore). 2015;94(18):e807. doi:10.1097/MD.0000000000000807

3. Oculocardiac Reflex. https://www.ncbi.nlm.nih.gov/pubmed/ 29763007. 
4. Arnold RW. The human heart rate response profiles to five vagal maneuvers. Yale J Biol Med. 1999;72(4):237-244.

5. Ha SG, Huh J, Lee BR, Kim SH. Surgical factors affecting oculocardiac reflex during strabismus surgery. BMC Ophthalmol. 2018;18 (1):103. doi:10.1186/s12886-018-0771-9

6. Ohashi T, Kase M, Yokoi M. Quantitative analysis of oculocardiac reflex by traction on human extraocular muscle. Invest Ophthalmol Vis Sci. 1986;27:1160-1164.

7. Machida CJ, Arnold RW. The effect of induced muscle tension and fatigue on the oculocardiac reflex. Binoc Vis and Strabismus Quart. 2003;18(2):81-86.

8. Arnold RW, Rinner AR, Arnold AW, Beerle BJ. The impact of re-operation, relatives and race on the oculocardiac reflex. Clin Ophthalmol. 2020;14:4253-4261. doi:10.2147/OPTH.S288578

9. Khurana I, Sharma R, Khurana AK. Experimental study of oculocardiac reflex (OCR) with graded stimuli. Indian J Physiol Pharmacol. 2006;50(2):152-156.

10. Yamashita M. Oculocardiac reflex and the anesthesiologist. Mid East $J$ Anesthesiol. 1986;8(5):399-415.

11. Bond AN, Beerle BJ, Arnold RW. Dexmedetomidine nasal sedation produces more oculocardiac reflex during strabismus surgery. J Pediatr Ophthalmol Strabismus. 2016;53(5):318. doi:10.3928/ 01913913-20160629-05

12. Arnold RW, Bond AN, McCall M, Lunoe L. The oculocardiac reflex and depth of anesthesia measured by brain wave. BMC Anesthesiol. 2019;19(1):36. doi:10.1186/s12871-019-0712-z

13. Arnold RW, Jensen PA, Kovtoun TA, Maurer SA, Schultz JA. The profound augmentation of the oculocardiac reflex by fast acting opioids. Binocul Vis Strabismus Q. 2004;19(4):215-222.

14. Arnold RW, Biggs RE, Beerle BJ. Intravenous dexmedetomidine augments the oculocardiac reflex. J AAPOS. 2018;22(3):211-213. e211. doi:10.1016/j.jaapos.2018.01.016

15. Arnold RW, Farah RS, Monroe G. The attenuating effect of intraglossal atropine on the oculocardiac reflex. Binocul Vis Strabismus $Q$. 2002;17(4):313-318

16. Fry E, Hall-Parker JB, Hall-Parker J. Eye hue and the oculocardiac reflex. Brit $J$ Ophthalmol. 1978;62:116-117. doi:10.1136/ bjo.62.2.116

17. Stump M, Arnold RW, Arnold RW. Iris color alone does not predict susceptibility to the oculocardiac reflex in strabismus surgery. Binocul Vis Strabismus Q. 1999;14(2):111-116.

18. Schumacher AC, Ball M, Arnold AW, et al. Oculocardiac reflex during ROP exams. Clin Ophthalmol. 2020;14:4263-4269. doi: $10.2147 /$ OPTH.S288043

19. Lai YH, Hsu HT, Wang HZ, Cheng KI, Wu K-Y. The oculocardiac reflex during strabismus surgery: its relationship to preoperative clinical eye findings and subsequent postoperative emesis. J AAPOS. 2014;18(2):151-155. doi:10.1016/j.jaapos.2013.11.024

20. Chung CJ, Lee JM, Choi SR, Lee SC, Lee JH. Effect of remifentanil on oculocardiac reflex in paediatric strabismus surgery. Acta Anaesthesiol Scand. 2008;52(9):1273-1277. doi:10.1111/j.13996576.2008.01745.x
21. Garg R. Remifentanil-induced bradycardia or oculocardiac reflex during strabismus surgery? Acta Anaesthesiol Scand. 2009;53 (4):548-549. doi:10.1111/j.1399-6576.2008.01898.x

22. Mizrak A, Erbagci I, Arici T, et al. Dexmedetomidine use during strabismus surgery in agitated children. Med Princ Pract. 2011;20 (5):427-432. doi:10.1159/000324554

23. Mizrak A, Erbagci I, Arici T, et al. Ketamine versus propofol for strabismus surgery in children. Clin Ophthalmol. 2010;4:673-679. doi:10.2147/opth.s11336

24. Liu Y, Zeng Q-Y. [Sevoflurane-N2O inhalation anaesthesia with laryngeal mask airway and propofol-ketamine intravenous anaesthesia in strabismus surgery]. Zhong Nan Da Xue Xue Bao Yi Xue Ban. 2006;31(1):97-99. Chinese.

25. Goerlich TM, Foja C, Olthoff D. [Effects of sevoflurane versus propofol on oculocardiac reflex-a comparative study in 180 children]. Anaesthesiol Reanim. 2000;25(1):17-21. German.

26. Snellen F, Vanacker B, Aken HV, VanAken H. Propofol-nitrous oxide versus thiopental sodium-isoflurane-nitrous oxide for strabismus surgery in children. J Clin Anesth. 1993;5:37-41. doi:10.1016/09528180(93)90085-S

27. Ducloyer JB, Couret C, Magne C, Lejus-Bourdeau C, Weber M, Le Meur G, Lebranchu P. Prospective evaluation of anesthetic protocols during pediatric ophthalmic surgery. Eur J Ophthalmol. 2018;1120672118804798.

28. Arnold RW, Wolfe T, Albrecht W, et al. Continuous monitoring and control of end-tidal anesthetic agent and $\mathrm{CO} 2$ mitigates the oculocardiac reflex during strabismus surgery. Binoc Vis Strabismus $Q$. 1996;11(4):281-288

29. Karaman T, Demir S, Dogru S, et al. The effect of anesthesia depth on the oculocardiac reflex in strabismus surgery. $J$ Clin Monit Comput. 2016;30(6):889-893. doi:10.1007/s10877-0159789-1

30. Yi C, Jee D. Influence of the anaesthetic depth on the inhibition of the oculocardiac reflex during sevoflurane anaesthesia for paediatric strabismus surgery. Br J Anaesth. 2008;101(2):234-238. doi:10.1093/bja/ aen129

31. Karanovic N, Jukic M, Carev M, Kardum G, Dogas Z. Rocuronium attenuates oculocardiac reflex during squint surgery in children anesthetized with halothane and nitrous oxide. Acta Anaesthesiol Scand. 2004;48(10):1301-1305. doi:10.1111/j.13996576.2004.00523.x

32. Loewinger J, Friedmann-Neiger I, Cohen M, Levi E. Effects of atracurium and pancuronium on the oculocardiac reflex in children. Anesth Analg. 1991;73:25-28. doi:10.1213/00000539-19910700000005
Clinical Ophthalmology

\section{Publish your work in this journal}

Clinical Ophthalmology is an international, peer-reviewed journal covering all subspecialties within ophthalmology. Key topics include: Optometry; Visual science; Pharmacology and drug therapy in eye diseases; Basic Sciences; Primary and Secondary eye care; Patient Safety and Quality of Care Improvements. This journal is indexed on PubMed
Central and CAS, and is the official journal of The Society of Clinical Ophthalmology (SCO). The manuscript management system is completely online and includes a very quick and fair peer-review system, which is all easy to use. Visit http://www.dovepress.com/ testimonials.php to read real quotes from published authors. 